\title{
La esteroidogénesis en el síndrome de ovarios poliquísticos
}

\author{
Estefanía Ventura-Arizmendi y Marcelino Hernández-Valencia
}

Instituto Mexicano del Seguro Social, Centro Médico Nacional Siglo XXI, Hospital de Especialidades, Unidad de Investigación Médica de Enfermedades Endocrinas, Ciudad de México, México

\begin{abstract}
Resumen
El síndrome de ovarios poliquísticos es la enfermedad endocrina más frecuente en la edad reproductiva; se caracteriza por alteraciones menstruales, hiperandrogenismo clínico o bioquímico e identificación ultrasonográfica de quistes ováricos. Las alteraciones neuroendocrinas y metabólicas que lo acompañan implican desensibilización del eje hipotálamo-hipófisis-ovario, esteroidogénesis e hiperandrogenismo. Recientemente se ha explorado el papel de la resistencia a la insulina. Se ha establecido que la principal causa del síndrome de ovarios poliquísticos es el hiperandrogenismo, debido a alteraciones enzimáticas en la vía esteroidogénica, por lo que existe sobreestimulación por parte de la hormona luteinizante a causa de los pulsos rápidos generados por la hormona liberadora de gonadotropinas. Diversos factores de crecimiento y citocinas inhiben la conversión de andrógenos a estrógenos. En la desregulación característica de este síndrome también están involucradas la activina y las prostaglandinas e, incluso, altos niveles de insulina.
\end{abstract}

PALABRAS CLAVE: Síndrome de ovarios poliquísticos. Esteroidogénesis. Hiperandrogenismo. Aromatización.

\begin{abstract}
Polycystic ovary syndrome is the most common endocrine disease in reproductive age, characterized by menstrual alterations, clinical or biochemical hyperandrogenism, and ultrasound-identified ovarian cysts. The neuroendocrine and metabolic alterations that accompany this condition involve the desensitization of the hypothalamus-pituitary-ovary axis, steroidogenesis and hyperandrogenism; recently, the role of insulin resistance has been explored. Hyperandrogenism has been established to be the main cause of polycystic ovary syndrome, due to enzymatic alterations in the steroidogenic pathway that cause luteinizing hormone over-stimulation because of quick pulses generated by gonadotropin-releasing hormones. Various growth factors of and cytokines inhibit the conversion of androgens into estrogens; activin and prostaglandins are also involved, even high levels of insulin participate in the characteristic deregulation of this syndrome.
\end{abstract}

KEY WORDS: Polycystic ovary syndrome. Steroidogenesis. Hyperandrogenism. Aromatization.

Fecha de recepción: 05-12-2017

Fecha de aceptación: 30-06-2018 DOI:10.24875/GMM.18003955
Gac Med Mex. 2019;155:184-190

Disponible en PubMed www.gacetamedicademexico.com 


\section{Introducción}

El síndrome de ovarios poliquísticos (SOP) es la enfermedad endocrina más común que afecta a las mujeres en edad reproductiva. La prevalencia se ha reportado entre 4 y $8 \%$. Para entender la enfermedad es preciso comprender el eje hipotálamo-hipófisis-ovario, la fisiología del ovario y la fisiopatología del SOP.

\section{Actividad del eje neuroendocrino}

Con la pubertad inicia la maduración del eje hipotálamo-hipófisis-ovario y la secreción de la hormona liberadora de gonadotropina $(\mathrm{GnRH}),{ }^{2}$ inhibida durante la infancia. La liberación de GnRH se lleva a cabo en forma de pulsos, de cuya frecuencia y amplitud depende la producción de las hormonas luteinizante (LH) y folículo estimulante (FSH), ${ }^{3}$ que estimulan las células de la teca y las de la granulosa del ovario, respectivamente; ${ }^{4}$ las primeras producen androstenediona mientras que las segundas aromatizan para la conversión a estradiol. El resultado es un cambio en la vía metabólica hacia estrógenos, el cual se expresa con crecimiento de las mamas y huesos y depósito de grasa tipo ginecoide. ${ }^{5}$ En las células de la granulosa se han estudiado otros péptidos que inducen la expresión de receptores de LH y las enzimas esteroidogénicas durante el desarrollo temprano de las células de la teca. ${ }^{6}$

Así, en las mujeres la producción de andrógenos por parte de la glándula suprarrenal es muy importante. ${ }^{7}$ Durante este periodo, las células corticales, a través de la hormona adrenocorticotrópica en la zona fascicular, también liberan grandes cantidades de andrógenos, como dehidroepiandrosterona (DHEA) y sulfato de DHEA, responsables del desarrollo de los vellos púbico y axilar y del acné. El incremento de los andrógenos de origen ovárico también facilita el desarrollo del crecimiento del vello púbico. ${ }^{5}$

Es así como existen dos fenómenos entrelazados: la esteroidogénesis y la foliculogénesis, dependientes del eje hipotálamo-hipófisis-ovario, ${ }^{8}$ el cual es orquestado por la red neuronal de las gonadotropinas, las cuales son liberadas desde las vesículas de las terminales eferentes de las neuronas en la eminencia media y el sistema portal hipofisario. La secreción de FSH promueve la expresión para receptores de LH, la estimulación inicial para el desarrollo folicular y la aromatización en las células de la granulosa de androstenediona a estradiol. La LH es reconocida por su participación en la fase lútea por promover la secreción de progesterona; además de su gran actividad en la fase folicular, es responsable de inducir la síntesis de andrógenos e iniciar la maduración del ovocito. La exposición continua de la hipófisis por la estimulación de las gonadotropinas causa desensibilización, por lo que la secreción de estas es suprimida como una vía de retroalimentación.

Recientemente se ha descrito que la maduración y la sensibilización de la actividad de $\mathrm{GnRH}$ depende de un polipéptido de 145 aminoácidos conocido como kisspeptina. El gen que codifica la kisspeptina (KISS1) ha sido localizado en el cromosoma 1. Las neuronas que expresan kisspeptina están presentes en el núcleo arcuato, el núcleo periventricular y el núcleo anteroventral periventricular en el ratón. Adicionalmente, en el área preóptica anterodorsal y el núcleo del lecho de la estría terminal. ${ }^{9}$

Se ha pensado que la GnRH y la kisspeptina en el hipotálamo son componentes cruciales del eje hipotálamo-hipófisis-ovario y mantienen la función reproductiva, estimulando el crecimiento folicular y la síntesis de esteroides sexuales. ${ }^{10}$ Se ha establecido que las señales de kisspeptina en el hipotálamo medio basal son responsables de la generación de pulsos de la $\mathrm{GnRH},{ }^{11}$ ya que la ausencia de kisspeptina condiciona un estado de inmadurez del eje hipotálamo-gónadas, donde, al bloquear su receptor GPR-54, se pierde la funcionalidad de esta vía, mientras que la adición del polipéptido kisspeptina restablece la funcionalidad y pulsos del eje. ${ }^{2}$

La mayoría de los neurotransmisores y neuromoduladores poseen propiedades exitatorias e inhibitorias dependiendo de ciertos factores, como composición del neurocircuito, estado del desarrollo corporal a lo largo de la vida y del ambiente hormonal. Se ha establecido que una de las causas del origen del SOP durante la pubertad es la irregularidad en la liberación de $\mathrm{GnRH}{ }^{2}$

Existe otro mecanismo de regulación neuroendocrina con efecto inhibidor o estimulador de la actividad hipotalámica, que actúa a través de los neurotransmisores como el ácido gammaaminobutírico (GABA), el neuropéptido Y (NPY) y la melatonina, que inhiben la actividad hipotalámica; o el glutamato, el ácido aspártico ( $N$-methyl- $d$-aspartic acid, NMDA), la norepinefrina y las células de la glía, que tienen actividad estimuladora. $^{13}$

\section{Esteroidogénesis ovárica}

La foliculogénesis normal depende de los andrógenos intraováricos para la síntesis de estradiol, como 


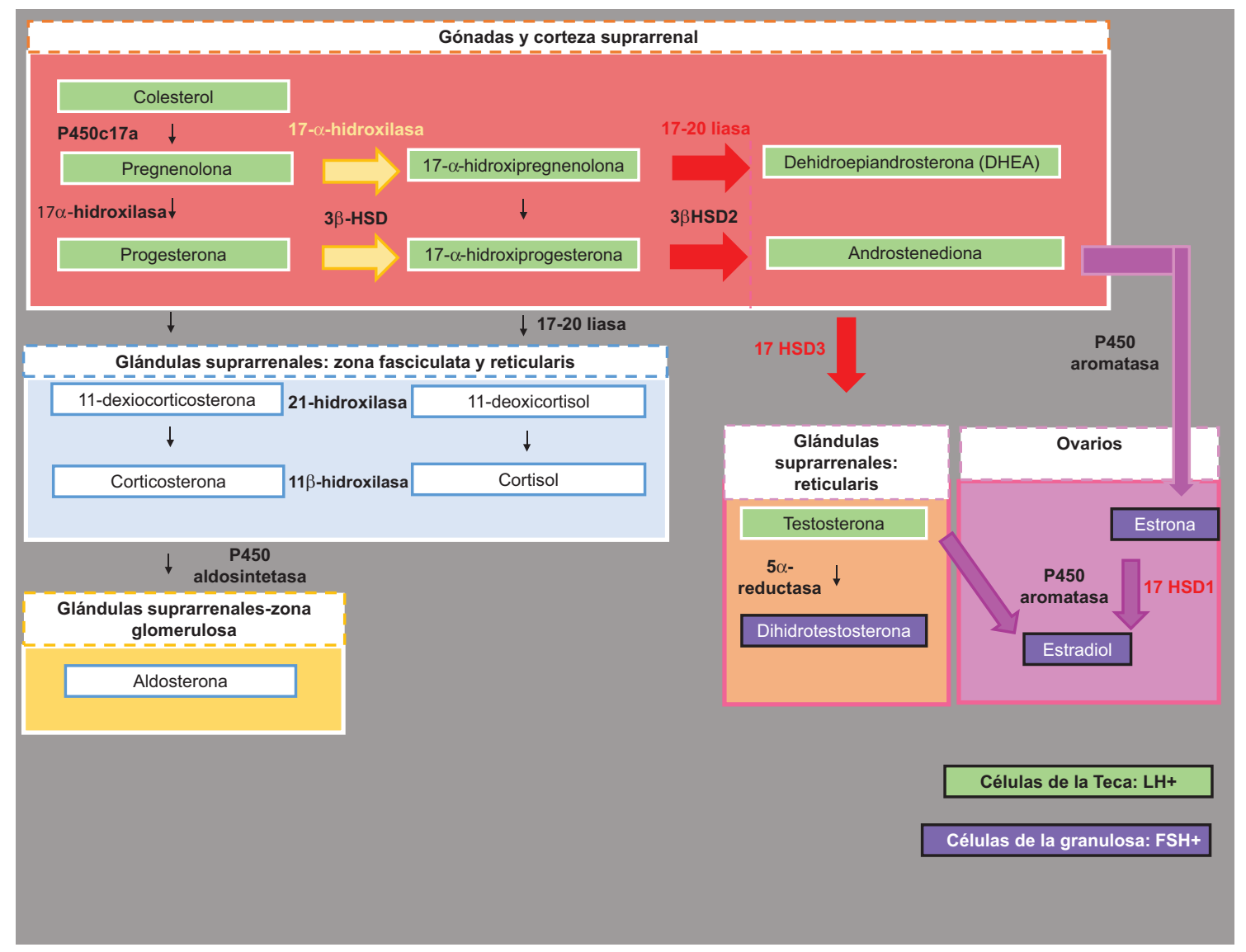

Figura 1. Esteroidogénesis ovárica y suprarrenal. Se muestran las vías metabólicas en los diferentes compartimentos glandulares. Cada compartimento cumple con la secreción de hormonas para este preciso balance. Las glándulas suprarrenales se dividen en corteza y médula (zona fasciculata, reticularis y glomurulosa). El recuadro rojo representa las gónadas y la corteza suprrarenal, donde el colesterol, precursor de esta vía metabólica, se transforma a través de una serie de pasos enzimáticos a progesterona y testosterona; a su vez, esta última en la zona reticularis se transforma libremente a dihidrotestosterona. El recuadro azul es la zona fasciculata y reticularis de la glándula suprrarenal donde se lleva a cabo la secreción de glucocorticoides como el cortisol, mientras que los mineralcorticoides (aldosterona) se representan en el cuadro amarillo. Finalmente ocurre la aromatización de los andrógenos a estradiol por medio de la P450 aromatasa en el ovario.

se ha descrito en la teoría "dos gonadotropinas-dos células". ${ }^{14}$ Esta teoría sobre la biosíntesis de los andrógenos refiere que las células de la teca secretan andrógenos en respuesta a la LH y que la androstenediona se transforma por la acción de la aromatasa a estrógenos en las células de la granulosa por influencia de la FSH, para lo cual debe existir un balance preciso. ${ }^{15}$ Así, el exceso en la producción de andrógenos es consecuencia de un desorden en el proceso de foliculogénesis, el cual se expresa con pobre maduración folicular e incremento en la atresia folicular. ${ }^{16}$

El paso inicial en la biosíntesis de todas las hormonas esteroideas es la conversión de colesterol a pregnenolona mediante dos vías que involucran la cadena de anclaje lateral de colesterol o la proteína reguladora modificable por la esteroidogénesis. Después, la pregnenolona es convertida en dehidroepiandroesterona a través de dos pasos dependientes de la enzima $\Delta 5$ esteroidea y la conversión es catalizada por el citocromo P450c17a. La progesterona experimenta una transformación paralela a androstenediona por la vía $\Delta 4$-esteroidea ( $\Delta 4-5$ isomerasa); al parecer, en el humano estas vías son más dependientes de la enzima 17-20 liasa para llegar por ambas síntesis a la androstenediona, antes de la actividad de la 17-cetoreductasa a testosterona o estradiol. En la glándula suprarrenal, la 17-hidroxiprogesterona es convertida en cortisol o en hormonas sexuales, dependiendo de si se utiliza la vía 21-hidroxilasa a cortisol o 17,20-liasa a 17-cetosteroides. La acción de 17- $\beta$-hidroxidehidrogenasa es esencial para la conversión de los 17-cetosteroides a testosterona, dihidrotestosterona y estradiol (Figura 1). ${ }^{15} \mathrm{La}$ LH actúa en el estroma intersticial de las células de la teca del ovario, induciendo la secreción de androstenediona, y posteriormente influye en la conversión de esta a estradiol por la acción de la aromatasa. ${ }^{17}$ 


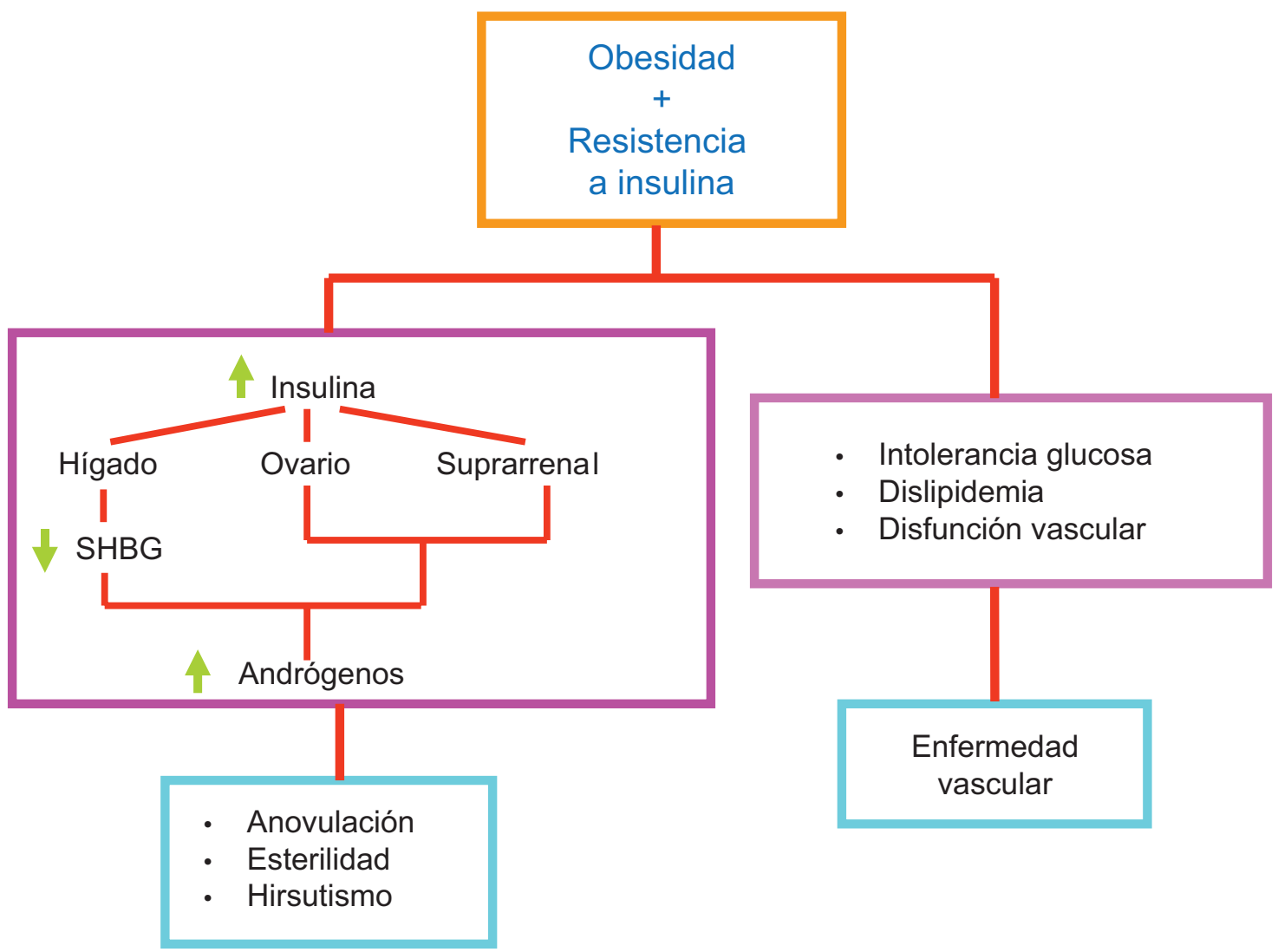

Figura 2. Fisiopatología del síndrome de ovario poliquísticos (SOP). Se describe el estado hiperinsulinémico que modifica la actividad hepática, ovárica y suprarrenal con las subsecuentes alteraciones hormonales sistémicas que llevan a los trastornos fenotípicos del SOP y a la esterilidad, así como a trastornos vasculares sistémicos.

El desarrollo del folículo dominante está asociado con incremento en los niveles de estradiol, el cual es dominante sobre la concentración de andrógenos, sin embargo, no es un mecanismo de retroalimentación negativa. La coordinación de las células de la teca y la función de las células de la granulosa, y la subsecuente síntesis de andrógenos, es finalmente organizada por una combinación de moduladores autocrinos, paracrinos y endocrinos. En la transición de folículo primordial a primario participan factores de crecimiento tales como GDF-9 (growth and differentiation factor-9) y BMP-15 (bone morphogenetic protein-15), que a través de la señalización paracrina actúan predominantemente sobre las células de la granulosa. ${ }^{18}$

\section{Esteroidogénesis en el SOP}

La evidencia apunta que el inicio del SOP se debe al aumento de andrógenos, resultado de la desregulación esteroidogénica; ${ }^{19}$ como consecuencia de este aumento existe respuesta estrogénica limitada. La sobreestimulación de LH, en tiempo y dosis dependiente, resultado de la regulación negativa de los receptores de $\mathrm{LH}$, reduce la actividad de anclaje del colesterol, la actividad de 17,20 liasa y de 17-hidroxilasa, con el subsecuente incremento en 17-hidroxiprogesterona a andrógenos..$^{20}$ En pacientes con SOP, la concentración de LH se encuentra elevada 40 a $60 \%$ comparada con la de población control, ${ }^{21}$ debido al aumento en la amplitud y frecuencia de los pulsos de LH. ${ }^{22}$

El desarrollo de las células de la granulosa, y de este modo el incremento de la actividad de la aromatasa, también determina la producción de andrógenos. Un folículo sano $\geq 8 \mathrm{~mm}$ de diámetro convierte eficientemente la androstenediona a estradiol. Por el contrario, folículos atrésicos o quísticos tienen alta proporción de androstenediona a estradiol.

La acción de FSH en las células de la granulosa determina el crecimiento de los folículos sanos hasta los $2.5 \mathrm{~mm}$ de diámetro, en parte mediado por el sistema IGF (insulin-like growth factors) y las concentraciones fisiológicas de insulina, que en conjunto estimulan la producción de estradiol. La proteína transportadora de IGF (IGF-binding proteins) inhibe la bioactividad de FSH y es marcadamente expresada 


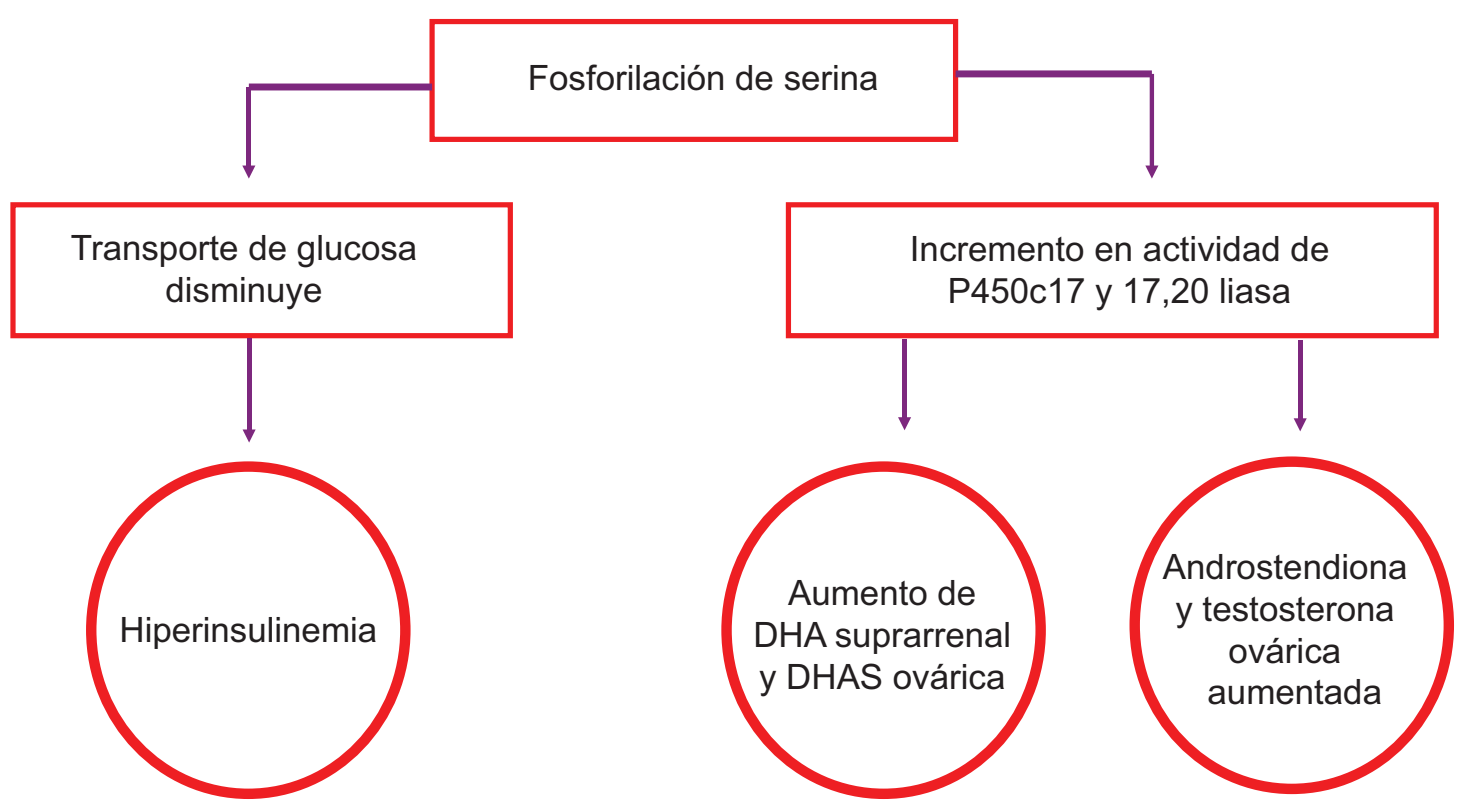

Figura 3. Defectos en señales de transducción. Los estados inflamatorios subclínicos y el estado metabólico alterado condicionan la fosforilación anormal de serina en el sustrato del receptor de insulina y, en consecuencia, el bloqueo de las vías metabólicas y enzimáticas que alteran todo el estado esteroidogénico.

en folículos atrésicos. La inhibina B es un péptido que recíprocamente está regulado por la FSH con una retroalimentación negativa, esencial para promover la síntesis de andrógenos desde las células de la teca, mientras que los mismos andrógenos sucesivamente estimulan la producción de esta, lo cual aún no es claro porque normalmente la FSH en forma predominante parece reducir los niveles de testosterona. ${ }^{23}$ Además, las prostanglandinas y la angiotensina también son promotoras, mientras que la hormona liberadora de corticotropinas y los factores de crecimiento- $\beta$, de crecimiento epidermoide, de necrosis tumoral y las citocinas tienen un papel inhibitorio en la biosíntesis de andrógenos. Lo anterior puede deberse a que los factores epidermoides y de crecimiento inhiben la aromatasa, mientras que la activina estimula a las células granulosas a producir estrógenos, al mismo tiempo que inhibe la secreción de andrógenos de la teca, por lo que cuando se pierde esta regulación predomina el estado hiperandrogénico característico del SOP. ${ }^{15}$

De esta forma, la insulina incrementa la estimulación de LH en la producción androgénica. Mientras que la FSH regula los siguientes pasos en el proceso de la foliculogénesis a través de la selección de folículo dominante, los andrógenos y estrógenos son modulares negativos del efecto de la $\mathrm{LH}$, ya que sinérgicamente el factor de crecimiento insulinoide tiene efecto positivo sobre el crecimiento folicular. ${ }^{15}$
La síntesis androgénica también está correlacionada con el desarrollo de las células de la granulosa y se asocia con la actividad de la aromatasa. La conversión eficiente de androstenediona a estradiol ocurre en folículos sanos $>8 \mathrm{~mm}$ de diámetro, por lo que este mecanismo es interrumpido por los folículos atrésicos y quísticos, lo que resulta en androstediona alta. Como consecuencia, la bioactividad de FSH es inhibida por las proteínas IGF, las cuales son expresadas significativamente en los folículos atrésicos. También los factores de crecimiento transformante y de fibroblastos inhiben la aromatasa y, consecuentemente, la síntesis de estradiol. ${ }^{15}$

Los pacientes con SOP tienen una tendencia al exceso de estradiol en todas las fases de maduración folicular. Se ha reportado que las células relacionadas con el SOP pierden respuesta a la FSH y producen menos cantidad de progesterona. ${ }^{24}$

El hiperandrogenismo suprarrenal es un tipo de disfunción androgénica caracterizada por hiperrespuesta de la DHEA e hipersensibilidad a la hormona adrenocorticotropa, sin embargo, estudios experimentales con análogos de $\mathrm{GnRH}$ han demostrado leve disminución, aunque significativa, de los andrógenos, contrario a lo que sucede con la dexametasona, con la que no hay cambios en las concentraciones de andrógenos, lo que sugiere que el origen de los andrógenos del SOP es ovárico, si bien este evento no ha quedado totalmente esclarecido. ${ }^{25}$ 


\section{Fisiopatología del SOP}

La fisiopatología del SOP es compleja porque en ella interactúan factores genéticos, metabólicos, fetales y ambientales. Las alteraciones predominantes son la secreción desordenada de gonadotropinas, hiperandrogenemia, resistencia a la insulina, hiperinsulinemia, disfunción ovárica e interrupción folicular (Figura 2). ${ }^{26}$ Una constante característica del SOP es el aumento de los niveles de LH, disminución o valores normales de FSH y persistente frecuencia rápida de pulsos en la secreción de la $\mathrm{GnRH} .{ }^{27}$ El papel del hiperandrogenismo en la regulación esteroidea de la $\mathrm{GnRH}$ y la secreción de gonadotropinas es descoordinado. La hipersecreción de LH afecta la síntesis ovárica de andrógenos, la foliculogénesis y el desarrollo del ovocito. ${ }^{28}$

El CYP17A1 (citocromo P450, familia 17, subfamilia $A$, miembro 1) es una monooxigenasa que se localiza en el retículo endoplasmático, la cual cataliza numerosas reacciones que involucran el metabolismo de diversas drogas, así como la síntesis de colesterol, esteroides y otros lípidos a través de al menos 10 enzimas que se encuentran en las mitocondrias de los diferentes tejidos con capacidad esteroidogénica como los ovarios, testículos, glándulas suprarrenales, placenta, adipocitos y algunas zonas del cerebro. Este citocromo cataliza 17-hidroxilasa y las reacciones subsecuentes de 17,20 liasa con pregnenolona, progestesterona y alopregnanolona. Por lo tanto, es una llave enzimática de la vía esteroidogénica para la producción de progestinas, mineralcorticoides, glucocorticoides, andrógenos y estrógenos. Las mutaciones de este gen están asociadas con la deficiencia del esteroide 17-a-hidroxilasa y 17-a-hidroxilasa/17,20-liasa, que causa pseudohermafroditismo e hiperplasia suprarrenal congénita. . $^{29,30}$

Diversos estudios indican que la hiperandrogenemia, en anovulación y ovulación en mujeres con SOP, se origina predominantemente en el ovario, además, confirman que la causa primaria de la producción excesiva de andrógenos en el SOP no está relacionada únicamente con la hipersecreción de $\mathrm{LH}$, por lo que se concluye que el defecto intrínseco está relacionado con la disfunción de células de la teca en el ovario $u$ otras influencias estimuladoras, como la insulina, IGF-1, entre otras citocinas. ${ }^{31}$

Se ha descrito que la LH actúa sobre las células de la granulosa en presencia de insulina y conduce a una luteinización temprana, interrupción del desarrollo folicular y exceso en la producción de andrógenos. ${ }^{24}$
En el desarrollo de la obesidad, la resistencia a la insulina está también relacionada con el factor de necrosis tumoral alfa, ya que aumenta la fosforilación de serina ${ }^{32}$ (Figura 3 ) en la vía de señales del receptor de insulina y sus sustratos, inhibiendo su señalización, y modifica el funcionamiento del ovario. ${ }^{7}$

\section{Consideraciones finales}

El SOP es la enfermedad endocrina más común que afecta a las mujeres en edad reproductiva. La información existente señala que es originado por una desviación en vía de la esteroidogénesis, con el consecuente aumento de los andrógenos, resultado de una desregulación hormonal en el ovario. Así, en el ovario los factores de crecimiento y epidermoides inhiben la aromatasa, mientras que la activina promueve que las células granulosas produzcan estrógenos, al mismo tiempo que inhibe la secreción de andrógenos de la teca, por lo que predomina el estado hiperandrogénico característico del SOP cuando se pierde dicha regulación.

\section{Bibliografía}

1. Sirmans SM, Pate KA. Epidemiology, diagnosis, and management of polycystic ovary syndrome. Clin Epidemiol. 2014;6:1-13.

2. Terasawa E, Fernandez DL. Neurobiological mechanisms of the onset of puberty in primates. Endocr Rev. 2001;1:111-151.

3. Besser GM, McNeilly AS, Anderson DC, Marshall JC, Harsoulis P, Hall R, et al. Hormonal responses to synthetic luteinizing hormone and follicle stimulating hormone-releasing hormone in man. Br Med J. 1972; 3:267-271.

4. Ciccone NA, Kaiser UB. The biology of gonadotroph regulation. Curr Opin Endocrinol Diabetes Obes. 2009;16:321-327

5. Roe AH, Dokras A. The diagnosis of polycystic ovary syndrome in adolescents. Rev Obstet Gynecol. 2011;4:45-51

6. Liu C, Peng J, Matzuk MM, Yao HH. Lineage specification of ovarian theca cells requires multicellular interactions via oocyte and granulosa cells. Nat Commun. 2015;6:6934-6955.

7. Baptiste CG, Battista MC, Trottier A, Baillargeon JP. Insulin and hyperandrogenism in women with polycystic ovary syndrome. J Steroid Biochem Mol Biol. 2010;122:42-52.

8. Baskind NE, Balen AH. Hypothalamic-pituitary, ovarian and adrenal contributions to polycystic ovary syndrome. Best Pract Res Clin Obstet Gynaecol. 2016;37:80-97.

9. Gianetti E, Seminara S. Kisspeptin and KISS1R: a critical pathway in the reproductive system. Reproduction. 2008;136:295-301.

10. Oride A, Kanasaki H, Mijiddorj T, Sukhbaatar U, Ishihara T, Kyo S. Regulation of kisspeptin and gonadotropin releasing hormone expression in rat placenta: study using primary cultures of rat placental cells. Reprod Biol Endocrinol 2015;13:90-98.

11. Ezzat A, Pereira A, Clarke IJ. Kisspeptin is a component of the pulse generator for $\mathrm{GnRH}$ secretion in female sheep but not the pulse generator. Endocrinology. 2015;156:1828-1837.

12. Hanchate NK, Parkash J, Bellefontaine N, Mazur D, Colledge WH, D'Anglemont-De-Tassigny $\mathrm{X}$, et al. Kisspeptin-GPR54 signaling in mouse NO-synthesizing neurons participates in the hypothalamic control of ovulation. J Neurosci. 2012;32:932-945.

13. Donoso AO, López FJ, Negro-Vilar A. Cross-talk between excitatory and inhibitory amino acids in the regulation of luteinizing hormone-releasing hormone secretion. Endocrinology. 1992;131:1559-1561.

14. Gougeon A. Regulation of ovarian follicular development in primates: facts and hypotheses. Endocr Rev. 1996;17:121-155.

15. Balen AH, Conway GS, Homburg R, Legro RS. Polycystic ovary syndrome. A guide to clinical management. EE. UU.: Taylor \& Francis; 2005.

16. Rosenfield RL, Ehrmann DA. The pathogenesis of polycystic ovary syndrome (PCOS): the hypothesis of PCOS as functional ovarian hyperandrogenism revisited. Endocr Rev. 2016;37:467-520. 
17. Barbieri RL. The endocrinology of the menstrual cycle. En: Rosenwaks Z, Wassarman PM, editores. Human Fertility. Methods and Protocols. EE. UU.: Humana Press; 2014.

18. Otsuka F, McTavish K, Shimasaki S. Integral role of GDF-9 and BMP-15 in ovarian function. Mol Reprod Dev. 2011;78:9-21.

19. Rosenfield RL, Barnes RB, Cara JF, Lucky AW. Dysregulation of cytochrome P450c17 alpha as the cause of polycystic ovarian syndrome. Fertil Steril. 1990;53:785-791.

20. White D, Leigh A, Wilson C, Donaldson A, Franks S. Gonadotrophin and gonadal steroid response to a single dose of a long-acting agonist of gonadotrophin-releasing hormone in ovulatory and anovulatory women with polycystic ovary syndrome. Clin Endocrinol (Oxf). 1995;42:475-481.

21. Taylor AE, McCourt B, Martin K, Anderson EJ, Adams J, Schoebfeld D, et al. Determinants of abnormal gonadotropin secretion in clinically defined women with polycystic ovary syndrome. J Clin Endocrinol Metab. 1997;82:2248-2256.

22. Barontini M, García-Rudaz M, Veldhius JD. Mechanisms of hypothalamic-pituitary-gonadal disruption in polycystic ovarian syndrome. Arch Med Res. 2001;32:544-552.

23. Hirshfeld-Cytron J, Barnes RB, Ehrmann DA, Caruso A, Mortensen MM, Rosenfield RL. Characterization of functionally typical and atypical types of polycystic ovary syndrome. J Clin Endocrinol Metab. 2009:94:1587-1594.

24. Mason HD, Willis DS, Beard RW, Winston RM, Margara R, Frank S Estradiol production by granulosa cells of normal and polycystic ovaries: relationship to menstrual cycle history and to concentrations of gonadotrophins and sex steroids in follicular fluid. J Clin Endocrinol Metab. 1994;79:1355-1360.
25. Rosenfield RL, Mortensen M, Wroblewski K, Littlejohn E, Ehrmann DA. Determination of the source of androgen excess in functionally atypical polycystic ovary syndrome by a short dexamethasone androgensuppression test and a low-dose ACTH test. Hum Reprod. 2011;26: 3138-3146.

26. Dumesic DA, Oberfield SE, Stener-Victorin E, Marshall JC, Laven JS, Legro RS. Scientific statement on the diagnostic criteria, epidemiology, pathophysiology, and molecular genetics of polycystic ovary syndrome. Endocr Rev. 2015,36:487-525.

27. Kazer RR, Kessel B, Yen SS. Circulating luteinizing hormone pulse frequency in women with polycystic ovary syndrome. J Clin Endocrinol Metab. 1987:65:233-236.

28. Qiao J, Feng HL. Extra-and intra-ovarian factors in polycystic ovary syndrome: impact on oocyte maturation and embryo developmental competence. Hum Reprod Update. 2011:17:17-33.

29. RefSeq: NCBI Reference Sequence Database [Sitio web]. EEUU: National Center for Biothechnology Information/National Library of Medicine; 2008.

30. Peng HM, Im SC, Pearl NM, Turcu AF, Rege J, Waskell L, et al. Cytochrome b5 activates the 17,20-lyase activity of human cytochrome P450 17A1 by increasing the coupling of NADPH consumption to androgen production. Biochemistry. 2016;55:4356-4365

31. Balen, A. The pathophysiology of polycystic ovary syndrome: trying to understand PCOS and its endocrinology. Best Pract Res Clin Obstet Gynaecol. 2004;18:685-706.

32. Dunaif $A$. Insulin resistance and the polycystic ovary syndrome: mechanisms and implication for pathogenesis. Endocr Rev. 1997;18:774-800. 